\title{
Preliminary Study of Rib Articulated Model based on Dynamic Fluoroscopy Images
}

\author{
Pierre-Frederic Villard ${ }^{a}$, Pierre Escamilla ${ }^{b, c}$, Erwan Kerrien ${ }^{a, b}$, Sebastien Gorges ${ }^{c}$, Yves \\ Trousset $^{c}$ and Marie-Odile Berger ${ }^{a, b}$ \\ ${ }^{a}$ Université de Lorraine, LORIA, UMR 7503, Nancy, France \\ ${ }^{b}$ Inria, France \\ ${ }^{c}$ General Electric Healthcare, Buc, France
}

\begin{abstract}
We present in this paper a preliminary study of rib motion tracking during Interventional Radiology (IR) fluoroscopy guided procedures. It consists in providing a physician with moving rib three-dimensional (3D) models projected in the fluoroscopy plane during a treatment. The strategy is to help to quickly recognize the target and the no-go areas i.e. the tumor and the organs to avoid.

The method consists in i) elaborating a kinematic model of each rib from a preoperative computerized tomography (CT) scan, ii) processing the on-line fluoroscopy image and iii) optimizing the parameters of the kinematic law such as the transformed 3D rib projected on the medical image plane fit well with the previously processed image.

The results show a visually good rib tracking that has been quantitatively validated by showing a periodic motion as well as a good synchronism between ribs.
\end{abstract}

Keywords: Respiration model, image driven simulation, fluoroscopy processing, articulated model

\section{INTRODUCTION}

Interventional Radiology (IR) consists in practicing minimally invasive surgery procedures while being guided by on-line medical imagery. IR specialists can thus perform tumor embolization without opening a patient.

In the case of fluoroscopy guidance the image quality is not good enough to properly guide the surgical gesture. In particular, soft tissues are hardly visible in X-ray images. Intra-arterial injection of contrast agent is used to visualize blood vessels, particularly those feeding the biopsy target, as well as the main vessels to avoid. However, such visibility cannot be maintained and, due to the motion of the supporting organ, contrast medium must be injected each time the surgeon wishes to have a snapshot of the vasculature.

In this paper, the targeted clinical procedure is the transcutaneous biopsy and treatment of hepatic tumors, performed with a needle inserted through the liver. Liver deformation is mainly due to respiration: the ribs move, which implies the diaphragm to deform, (de-)compressing the liver. This chain reaction can be simulated: The global strategy is to track the respiration (intercostal) muscles, or equivalently the ribs, and the diaphragm in order to apply mechanical constraints on the liver to deform it. Two-dimensional (2D) fluoroscopy images could then be augmented with three-dimensional (3D) models of tumor and liver vascularization deforming in real time.

As a first step we propose here to concentrate on the ribs, and track their 3D motion in a single anteroposterior (AP) view acquired by real-time fluoroscopy, through the maximization of a $3 \mathrm{D} / 2 \mathrm{D}$ similarity criterion between the rib model and the fluoroscopy image.

The remainder of this paper is organized as follows: a state of the art is presented in the coming section, then the different steps of our method are presented in the next section. Finally the last section presents the various experiments we performed.

Further author information: Send correspondence to Pierre-Frederic Villard : E-mail: pierrefrederic.villard@loria.fr 


\section{PREVIOUS WORKS}

Tracking respiration motion has been widely studied in the past. Most of the works focused on radiotherapy applications where a precise knowledge of the tumor position is mandatory before practicing a highly invasive treatment. A recent state of the art about motion measurement in this context is presented in Bowen et $a l .{ }^{1}$ Solutions include body surface tracking with structured light, ${ }^{2}$ stereovision with square physical markers, ${ }^{3}$ reflective marker blocks, ${ }^{4}$ invasive markers ${ }^{5}$ and spirometer-guided non-rigid registration. ${ }^{6}$ Even if techniques presented work quite well in the ionizing therapy context it cannot be directly applied to IR procedures for two reasons. First the equipment as well as the involved protocols are too heavy to be used as a daily IR routine. Secondly most of these methods are based on the reproducibility of the respiration cycle and on a steady influence of the various respiration muscles. While it may be true during a controlled tidal breathing, it is too hazardous ${ }^{7}$ for IR procedures. For these reasons, we propose to resort to $3 \mathrm{D} / 2 \mathrm{D}$ registration techniques for rib tracking using a $3 \mathrm{D}$ articulated model of the rib.

The problem of $3 \mathrm{D} / 2 \mathrm{D}$ registration in image guided interventions has been largely addressed in the medical community. A recent review of such techniques can be found in Markelj et al. ${ }^{8}$ This review revealed that the field of $3 \mathrm{D} / 2 \mathrm{D}$ registration is dominated by DRR-based methods. Digitally Reconstructed Radiographs (DRR) are computed by projecting a CT scan from a preselected position and can be regarded as synthetic X-ray images. Registration is then achieved by maximizing an alignment score between the DRR and the current image. ${ }^{9}$ But most previous works restrained to the rigid case and non-rigid registration was hardly addressed. In the context of rib tracking, Schweikard et al. ${ }^{10}$ use two CT scans at inhale and exhale positions. A series of intermediate CT scans was computed by interpolating between both CT scans. Registration was thereafter obtained by identifying the DRR in the series, and thus a time step within the respiratory cycle, which was the closest to a live shot. A drawback of this method is that the respiratory model is computed by linear interpolation between two scans, which is not appropriate to account for the complexity of respiration induced deformations. Another disadvantage is the computational cost of the method which requires fine sampling if accuracy is needed. The situation worsens when elastic deformations are considered. The increase of the parameter space dimensionality forbids precomputing the deformed scans. Moreover inferring a physically coherent deformation of the whole scan from the rib model is not straightforward. Indeed, the ribs motion follows that of an articulated model.

Assuming a prior segmentation of the ribs in a pre-operative CT scan, we face a surface to image registration problem. For smooth surfaces without noticeable texture as the ribs, point correspondences may not be easily established. In this case the apparent or silhouette contour of the surface is the only feature available. ${ }^{11}$ The apparent contour is the projection of the locus of points which separates the visible from the occluded parts of an object. Though apparent contours are view-dependent and do not physically match, past works on rib tracking mostly considered rib contours as classical image contours. Tracking was guided by 2D motion hypothesis on the rib motion. Existing methods include contour line extraction based on: Hough transform, ${ }^{12}$ B-Spline defined by manually-picked control points and moving with a rigid registration, ${ }^{13}$ segmentation extracted from the knowledge of surrounding organs. ${ }^{14}$ In order to improve the robustness of the method, Zhang et al. ${ }^{15}$ proposed to use motion compensation to predict the position of the ribs as well as polynomial models of rib contours to improve detection. However, fine tuning of several thresholds was required to obtain correct results. In these past papers, difficulties in rib tracking originated in the low signal to loise ratio in low dose X-ray images as well as in the difficulty to model in $2 \mathrm{D}$ the projection of a 3D motion. The method proposed here originates in $3 \mathrm{D} / 2 \mathrm{D}$ registration techniques and in DRR-based methods but is based on an articulated model of the ribs. Parameters that govern the articulated model are optimized so as to fit the rib contours with the image. Instead of comparing the DRR image with the live image, we compare the apparent contours of the ribs model with the gradient in the live image.

\section{METHODS}

Following rib motion on the fluoroscopy image where quality is not as good as standard radiography is a difficult task. Our contribution is a method to extract rib kinematic laws customized to a patient. The method is based on a 3D articulated model which projection on a plane is optimized to satisfy a similarity criterion. This section presents in detail the main technical components that have been addressed. Our study uses two medical data: a computerized tomography (CT) scan and a fluoroscopy (see §3.1). The CT scan is used to construct 
the 3D model by manual segmentation and meshing and is also used to compute the rotation parameters using the eigenvectors of the inertial moments (see §3.2). The dynamic fluoroscopy is used for gradient extraction preprocessing to guide the rib transformation after this latter one has been registered in the same reference system (see §3.3). An optimization algorithm then computes the best transformation to define the rib kinematic law (see §3.4). The general workflow of our simulation is presented on Fig. 1. The general strategy is to find the angles of the articulated model and keep the best match with the fluoroscopy input image.

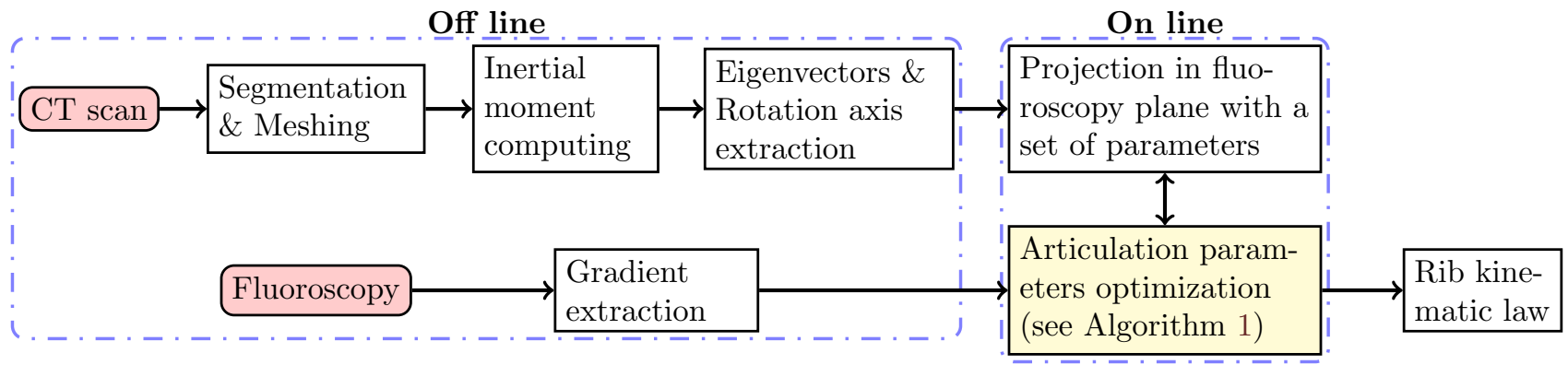

Figure 1. General overview of the method showing all the processing steps from the medical images to the rib kinematic law.

\subsection{Input Data}

A 3D CT scan and a dynamic fluoroscopy of a patient were selected. CT scan parameters were as follows: size $512 \times 512 \times 231$, and voxel dimensions are $0.76 \mathrm{~mm} \times 0.76 \mathrm{~mm} \times 1.25 \mathrm{~mm}$. Fluoroscopy dynamic imagery consists here of 300 time steps of images with a $1024 \times 1024$ resolution at a frame rate of 30 images per second. The CT scan has been manually segmented by a medical expert to determine the spine, the ribs and the diaphragm contours. Triangle meshes have been extracted using the Marching Cube algorithm. The geometries have then been simplified by smoothing and decimation.

\subsection{Rib rotation parameters extraction}

Rib motion could be assumed to be only a rigid kinematic motion. For instance, Didier et al. ${ }^{16}$ used a finite helical axis method i.e. composed of a translation and a rotation along an axis. We chose two rotations along two axis according to a medical study: ${ }^{17}$ the "bucket handle" rotation and the "pump handle" rotation. The parameters are for each rib: a rotation center, two rotation axis and two rotation angles associated with a time step. In order to extract those parameters we initially computed the moments of inertia of each rib and the associated eigenvectors. The eigenvectors of the inertia tensor define the principal axes of a rigid body. They are extracted from the rib plane. The intersection between this plane and the spine axis gives the rotation center. Figure 2.Left shows an example of ribs represented as a triangular mesh. The eigenvectors resulting from the previously presented method are the dark blue lines. Axis $\mathbf{A}$ and $\mathbf{B}$ are selected to define the rib plane. They are used to model the rotational motion similarly to Wilson et al. t. ${ }^{17}$ Rotational parameters are independently defined for each rib; and are composed of a rotation center and two rotation axes. The first axis $\mathbf{A}$ defines the "bucket handle" rotation (angle $\alpha$ ) and the second axis B defines the "pump handle" rotation (angle $\beta$ ). We obtain an articulated model of the ribs defined by constant and variable parameters (two angles $\alpha$ and $\beta$, see Fig.5.b). The next step is to optimized $\alpha$ and $\beta$ for each rib independently with on-line per-operative data. The aim is to monitor this model with image processing based criteria extracted here from each dynamic $2 \mathrm{D}$ fluoroscopy image.

\subsection{Fluoroscopy image preprocessing and Rib angle initialization}

The first step is to determine the position of the ribs on the first fluoroscopy image.

An initial projection matrix between the CT and the fluoroscopy can be determined using the system parameters contained in the DICOM file of the fluoroscopic image. The initial parameters are manually determined by tuning the $\alpha$ and $\beta$ parameters so that the projection of each rib fits at best with the first fluoroscopic image of the sequence (Cf Fig. 9 a. and b.). 

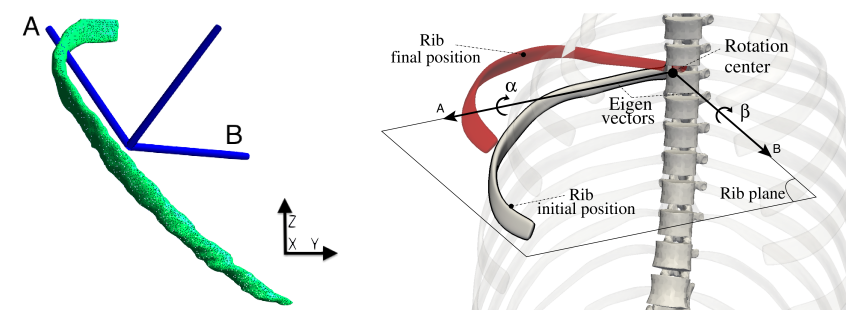

Figure 2. Left: rotation axes visualization of rib X in YZ plane. Right: Rib rotation algorithm. Inputs are: (i) Eigenvectors, (ii) rotation angles $\alpha$ and $\beta$, (iii) rotation center and (iv) initial rib location. The output is the rib at its final position.
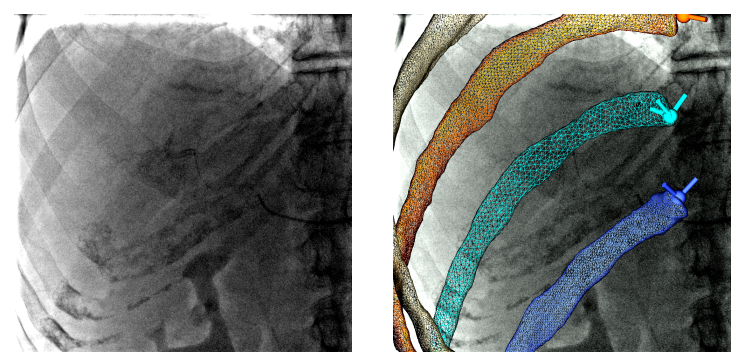

Figure 3. Fluoroscopic image. Left: fluoroscopy, right: 3D projection on the fluoroscopy plane with ribs 9 , 10,11 and 12 with center and axis for the rotation.

We apply then a gradient filter on the fluoroscopy image. All the 3D bone meshes are projected on the fluoroscopy image. The projection matrix Proj is found manually. The first time step of the initial image is displayed on Figure 3.left. Figure 3.right shows the projection of the meshes on the fluoroscopy at the initial time step. Each rib rotation parameter $(\alpha$ and $\beta)$ is manually tuned so the projection of the rib mesh matches well with the fluoroscopy. In our study we have 4 ribs to optimize because they are the ones visible in the fluoroscopy. $R_{n}(t)$ represents the mesh for rib $\# n$ at time step $t . R_{n}(0)$ - the mesh at the initial position - is extracted from the $\mathrm{CT}$ and is expressed in the $\mathrm{CT}$ reference system.

\subsection{Rib motion optimization}

Ribs being optimized one by one we focus here on one rib. Contrary to the initial fluoroscopy image, the other time step rotation parameters are automatically computed. The main idea is to compute the rib parameters $\alpha$ and $\beta$ by minimizing the distance between the occluding contours of the rib and the contours in the fluoroscopy image. The optimization stage is initialized with the parameters computed at the previous time-step. In the following the transformation of rib \#n between times 0 and $t$ - corresponding to the two rotations described in $\S 3.2$ - is called $T\left(\alpha_{t}, \beta_{t}\right)$, the projection matrix from the CT scan to the fluoroscopy reference system is still Proj, the image gradient is noted $\nabla$. Occluding contours are obtained by first computing the binary image - the occluding mask - of the projection of the rib (Cf Fig. 4.c) and then computing the boundaries of the mask (Cf Fig. 4.d) and the function to obtain the occluding mask is Proj $_{b i n}$ whose gradient gives the occluding contours.

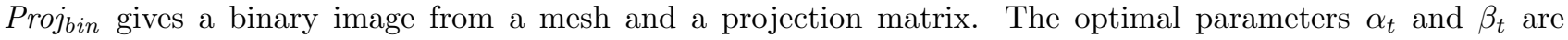
recovered so as to maximize the similarity between the occluding contours and the contours extracted in each fluoroscopy image. This optimization process is centered on Powell's minimization and is detailed in algorithm 1. Particularly, Fig. 4 shows illustrations of lines 7 and 9 of the algorithm. The optimization aims at maximizing 
the value of the similarity criterion (Cf Fig. 4.e) and converges when this maximum quantity gets stable.

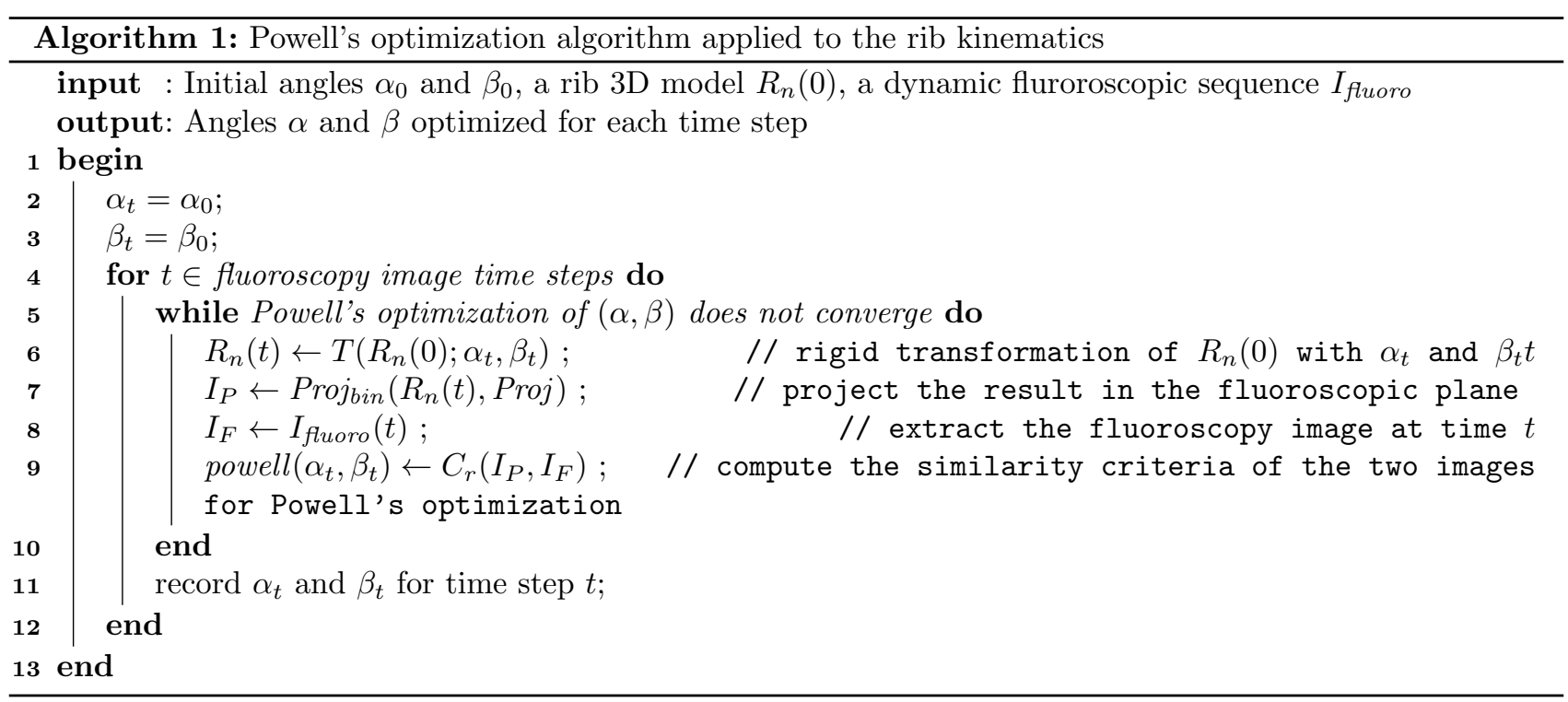

The computing time is higher in our case compared to the studies in the X-Ray context studied in $\S 2$ because the whole mesh surface is projected to compute the occluding contour.

$a$.

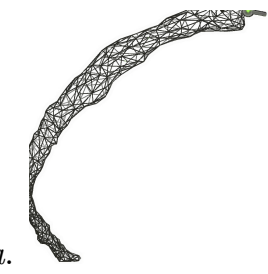

b.

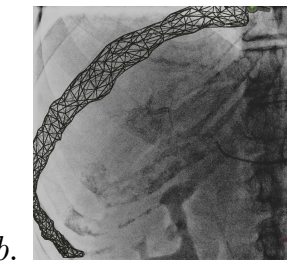

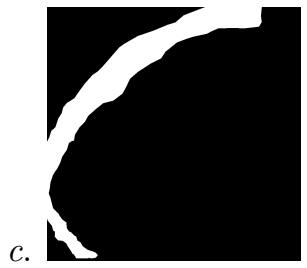

$d$.

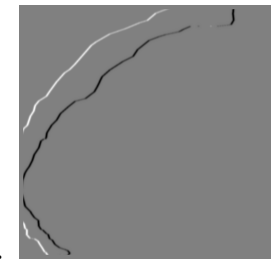

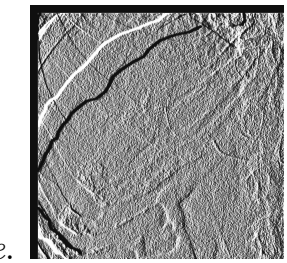

Figure 4. Similarity computation for a given rib rotation. a: rib $3 \mathrm{D}$ mesh, b: 3D mesh on the fluoroscopy, c: rib projection, d: edge computation, e: Composition of gradient intensity image and projected rib gradient

Finding a good similarity criterion between the projected model $\left(I_{P}\right)$ and the live fluoroscopy image $\left(I_{F}\right)$ is not a trivial task. Due to the lack of texture in the images of the rib, we here attempt to maximize the similarity between the apparent contours and the contours extracted in the fluoroscopy images. Note that in order to improve the quality of the fluoroscopy images, a Perona-Malik anisotropic diffusion filter ${ }^{18}$ is applied before performing optimization. We present here two solutions we have tested. In a first attempt, we used a classical correlation criterion based on the amplitude of the gradient. Sobel filter was applied on the fluoroscopic image $I_{F}$.The first similarity criteria $C r_{1}$ was then given by a sum on every pixel pix:

$$
C r_{1}\left(I_{P}, I_{F}\right)=\sum_{p i x} \| \nabla I_{P}(\text { pix })\|\cdot\| \nabla I_{F}(\text { pix }) \|
$$

This criterion encourages high gradients of the fluoroscopy images to be close to the apparent contours. In order to handle both imprecisions in the 3D model and the low signal to noise ratio, the occluding mask was smoothed so as to produce non-zero gradients in a small neighborhood of the contour points. Unfortunately, depending on the initialization, it might happen that the external border of one rib in the model converged towards the internal border of another rib in the fluoroscopy image. This is obviously due to the fact that the criterion only considers the amplitude of the gradient and not the direction. We thus propose a second criteria $\mathrm{Cr}_{2}$ based on the scalar product of gradient vectors:

$$
C r_{2}\left(I_{P}, I_{F}\right)=\sum_{p i x} \nabla I_{P}^{\top}(p i x) \cdot \nabla I_{F}(p i x)=\sum_{p i x} \frac{\partial I_{P}(p i x)}{\partial x} \cdot \frac{\partial I_{F}(p i x)}{\partial x}+\frac{\partial I_{P}(p i x)}{\partial y} \cdot \frac{\partial I_{F}(p i x)}{\partial y}
$$


This way, the internal and the external borders of the ribs cannot be confused since the gradients have opposite directions at these points. Fig.4.d is the horizontal gradient and Fig.4.e. its combination with the image gradient in the same direction.

\section{RESULTS AND DISCUSSIONS}

\subsection{Implementation}

The framework has been implemented in $\mathrm{C}++$ with the use of OpenScengraph* to handle 3D object rigid transformation and 3D rendering geometry, gts library ${ }^{\dagger}$ to manipulate the mesh models, QT library ${ }^{\ddagger}$ to design the graphical user interface and numerical recipes algorithm implementation.

The results below were obtained on a Mac Book Pro with 2Go RAM.

In order to have an initial idea of the expected signal linked to the respiration cycle, we plot the diaphragm motion. The diaphragm has an almost translation motion in the craniocaudal direction often compared to a pump motion. A craniocaudal line has been chosen and the diaphragm intersection with this line gives a point that represents the respiration cycle (Cf Fig.5.a). Fig.5.b show the resulting point displacement with respect to the time. The diaphragm signal is called Diaph. A periodic breathing cycle is recognizable and there were around six respiration cycles during the nine seconds of the experiment giving a respiration rate between 30 and 40.
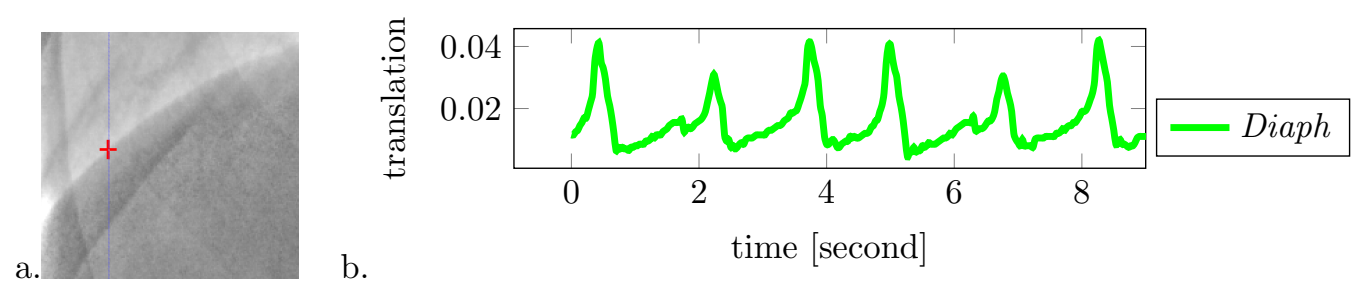

Figure 5. Point manually tracked on the diaphragm: intersection of a chosen craniocaudal line (in blue) and the diaphragm

\subsection{Correlation by gradient amplitude}

The initial validation tests we performed were with gradient amplitude correlation (criterion $C r_{1}$, equation (1)). The optimization process has been performed on four ribs. For each of them we plotted the $\alpha$ and $\beta$ angle evolution with respect with each time step of the fluoroscopy image. Fig. 9.a shows the results of the ninth rib in correspondence with the diaphragm motion signal. The results look like a staircase function where the values are steady on time intervals. The explanation is given by Fig. 6 showing the rib automatically positioned after Powell's optimization at various time step. These images have been extracted at first steps of Fig. 7 staircase function (after the jumps that occurred at 0.5, 0.8, 0.95 and $1.2 \mathrm{~s}$ ). The jumps from a level to another is explained by the fact that gradient amplitude image makes no difference between the outside and the inside of a rib. In order to avoid this kind of jump a solution is to consider instead gradient direction as implemented in $\mathrm{Cr}_{2}$.

\subsection{Correlation by gradient vector}

Gradient vectors are here computed for the initial fluoroscopy $\left(\frac{\partial I_{F}}{\partial x}\right.$ and $\left.\frac{\partial I_{F}}{\partial y}\right)$ and for each projection configuration $\left(\frac{\partial I_{P}}{\partial x}\right.$ and $\left.\frac{\partial I_{P}}{\partial y}\right)$ as shown on Fig. 8. Similarly to the previous section we plotted the $\alpha$ and $\beta$ variations during each time frame of the fluoroscopy for the same ninth rib with the criterion $C r_{2}$ given by equation (2). The result is presented on Fig. 9.left. The six breathing phases are synchronized. In order to quantify it we analyzed the signal with a Fourier power transform. There is a pronounced pike at about $30 \mathrm{~Hz}$ which is the respiration rate we anticipated. Fig.10 shows some images of the fluoroscopy sequence with the four ribs tracked with our articulated model.

\footnotetext{
*http://www.openscenegraph.org/ (last accessed: 20/01/2014)

${ }^{\dagger}$ http://gts.sourceforge.net/ (last accessed: 20/01/2014)

${ }^{\ddagger}$ http://qt-project.org/ (last accessed: 20/01/2014)
} 

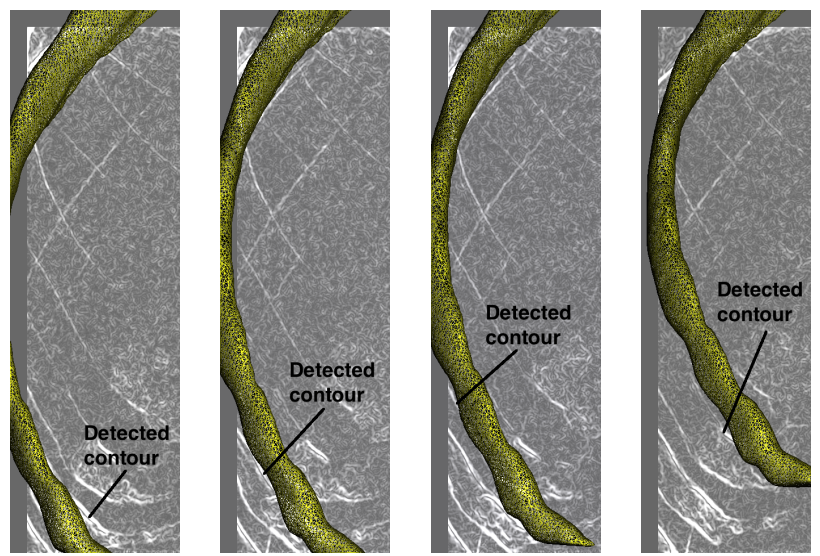

Figure 6. Rib rotation evolution: four time steps with different detected contours ( $\beta$ angle around $-0.07,0.015,0.05$ and 0.08 radian)

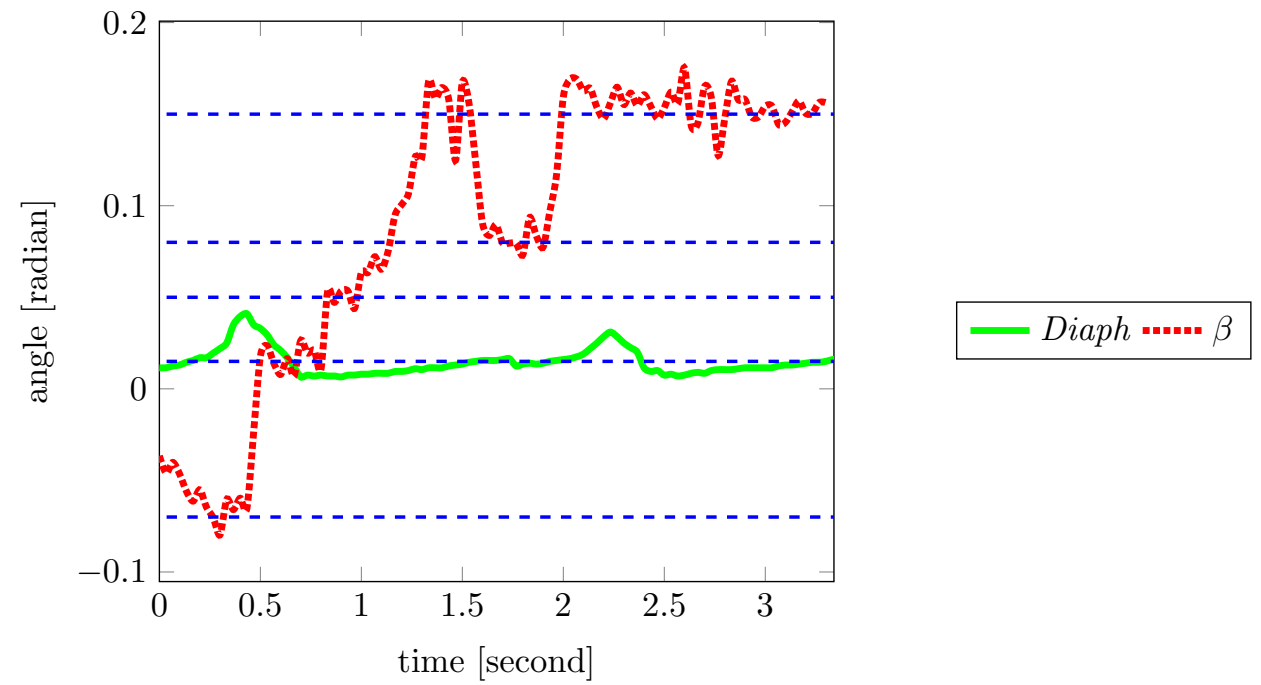

Figure 7. Angle rotation plot : different levels of value illustrated with dashed blue lines

\section{CONCLUSION}

We have presented here a method to track each rib separately on a fluoroscopic image with respect to the respiration motion. The idea is to use this information in IR procedures to guide the physician during the treatment. Our main contribution is an articulated model of the ribs. After the initialization step, the method is fully automatic without any use of other tunable parameters as in other models of the literature. Each rib behavior is computed with an optimization of the rigid transformation based on the similarity of the projected rib with the fluoroscopy reference image. With respect to existing methods based on respiratory simulation, ${ }^{10}$ an advantage of our technique is that it does not require to simulate the elastic deformation of the complete scan during breathing - i.e ribs + surrounding organs-. Indeed, an articulated model of the rib is acknowledged to be a good and realistic model of the rib motion whereas generating realistic elastic deformation of the scan is difficult and patient-dependent. The method has been tested on a real patient database. The results show a good periodicity as well as a good synchronization of the signal. In the future we plan to add a constraint to link the ribs together as well as to use precomputed Digitally Reconstructed Radiography to decrease the computing time. In longer term we plan to also model the diaphragm in order to eventually monitor the liver deformation still with image guided information. 

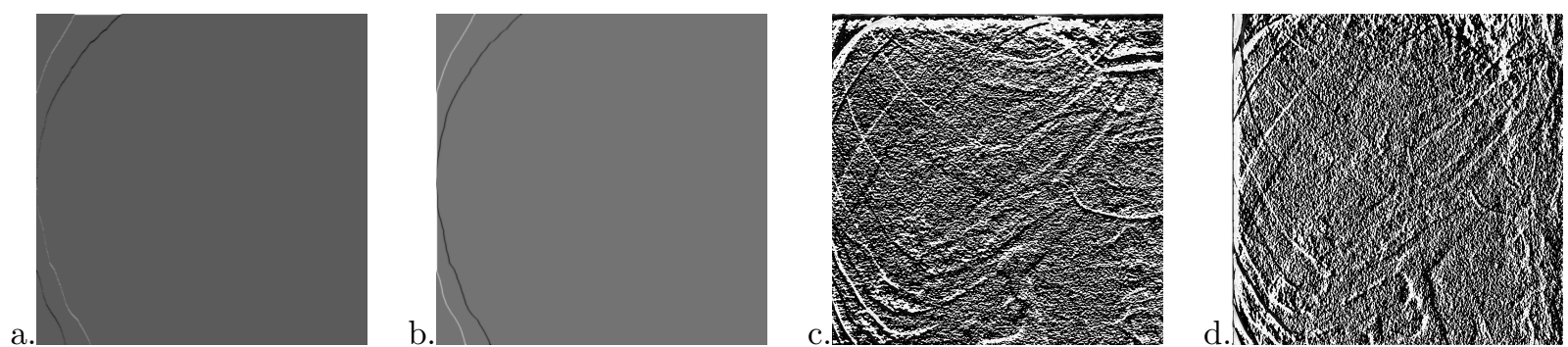

Figure 8. Gradient vector results. a: projected ribs $\frac{\partial I_{P}}{\partial x}$; b: projected ribs $\frac{\partial I_{P}}{\partial y}$; c: fluoroscopy $\frac{\partial I_{F}}{\partial x}$ and d: fluoroscopy $\frac{\partial I_{F}}{\partial y}$
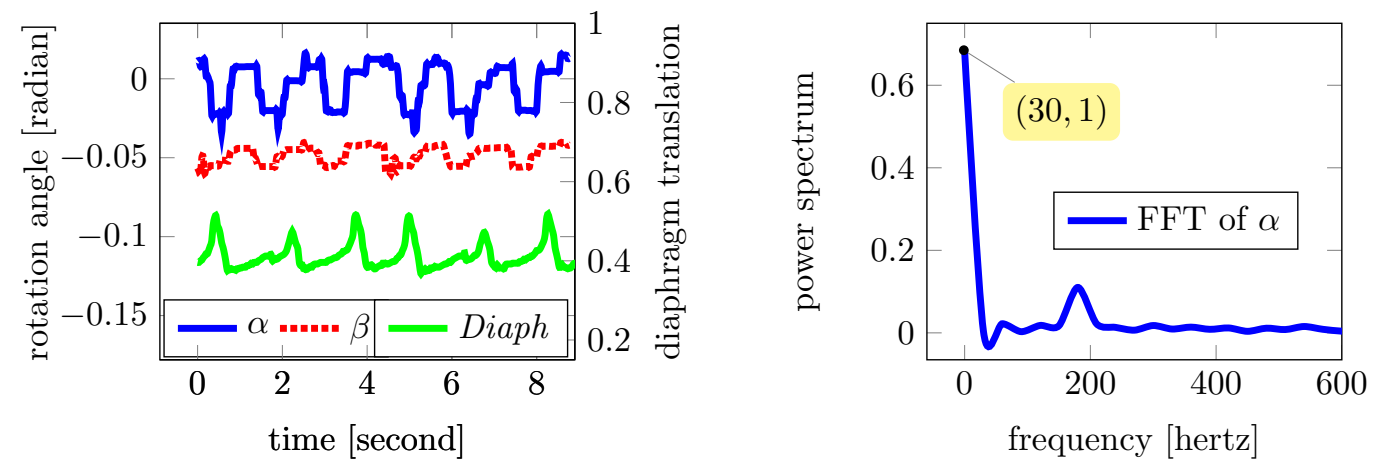

Figure 9. a: Respiration signal for rib angles and diaphragm motion, b: Rib rotation algorithm. Inputs are: (i) Eigenvectors, (ii) rotation angles $\alpha$ and $\beta$, (iii) rotation center and (iv) initial rib location.

\section{REFERENCES}

[1] Bowen, S. R., Nyflot, M. J., Gensheimer, M., Hendrickson, K. R. G., Kinahan, P. E., Sandison, G. A., and Patel, S. A., "Challenges and opportunities in patient-specific, motion-managed and pet/ct-guided radiation therapy of lung cancer: review and perspective," Clin Transl Med 1(1), 18 (2012).

[2] Parkhurst, J. M., Price, G. J., Sharrock, P. J., Marchant, T. E., and Moore, C. J., "Next generation optical surface sensing for real-time measurement in radiotherapy," Conf Proc IEEE Eng Med Biol Soc 2011, 6170-3 (2011).

[3] Hostettler, A., Nicolau, S., Forest, C., Soler, L., and Remond, Y., "Real time simulation of organ motions induced by breathing: First evaluation on patient data," Biomedical Simulation 1, 9-18 (2006).

[4] Putra, D., Haas, O. C. L., Mills, J. A., and Burnham, K. J., "A multiple model approach to respiratory motion prediction for real-time igrt," Phys Med Biol 53, 1651-63 (Mar 2008).

[5] Nuyttens, J. J., Prevost, J.-B., Praag, J., Hoogeman, M., Van Klaveren, R. J., Levendag, P. C., and Pattynama, P. M. T., "Lung tumor tracking during stereotactic radiotherapy treatment with the cyberknife: Marker placement and early results.," Acta Oncol 45(7), 961-5 (2006).

[6] Sarrut, D., Boldea, V., Miguet, S., and Ginestet, C., "Simulation of four-dimensional ct images from deformable registration between inhale and exhale breath-hold ct scans," Medical physics 33, 605 (2006).

[7] Shirato, H., Suzuki, K., Sharp, G. C., Fujita, K., Onimaru, R., Fujino, M., Kato, N., Osaka, Y., Kinoshita, R., Taguchi, H., et al., "Speed and amplitude of lung tumor motion precisely detected in four-dimensional setup and in real-time tumor-tracking radiotherapy," International Journal of Radiation Oncology* Biology* Physics 64(4), 1229-1236 (2006).

[8] Markelj, P., Tomazevic, D., Likar, B., and Pernus, F., "A review of 3d/2d registration methods for image-guided interventions," Medical Image Analysis 16(3), 642-661 (2012).

[9] Penney, G., Weese, J., Little, J., Desmedt, P., Hill, D., and Hawkes, D., "A comparison of similarity measures for use in 2-d-3-d medical image registration," Medical Imaging, IEEE Transactions on 17(4), 586-595 (1998).

[10] Schweikard, A., Shiomi, H., and Adler, J. R., "Respiration tracking in radiosurgery without fiducials," Medical Robotics and Computed Assisted Surgery 1(2), 19-27 (2005).

[11] Cipolla, R. and Blake, A., "Surface shape from the deformation of apparent contours," International Journal of Computer Vision 9(2), 83-112 (1992). 

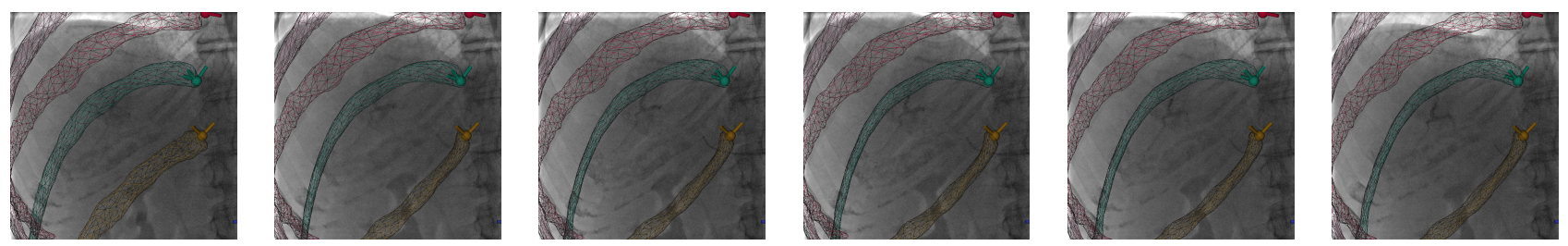

Figure 10. Sequence of augmented fluroroscopy with the four ribs

[12] Yue, Z., Goshtasby, A., and Ackerman, L. V., "Automatic detection of rib borders in chest radiographs," Medical Imaging, IEEE Transactions on 14(3), 525-536 (1995).

[13] Myint, S. M., Komizu, M., Yanagihara, Y., and Hama, H., "Analyzing the 3d shape and respiratory motion of the ribs using chest x-ray images," Medical imaging technology 20, 694-702 (nov 2002).

[14] Sarkar, S. and Chaudhuri, S., "Detection of rib shadows in digital chest radiographs," in [Image Analysis and Processing], 356-363, Springer (1997).

[15] Zhang, H., Ruan, X., Komizu, M., Yanagihara, Y., and Hama, H., "Tracing rib contour lines by respiratory motion compensation from an x-ray image sequence," Medical Imaging Technology. 22(3), 143-154 (2004).

[16] Didier, A.-L., Villard, P.-F., Bayle, J.-Y., Beuve, M., and Shariat, B., "Breathing thorax simulation based on pleura physiology and rib kinematics," in [Medical Information Visualisation-BioMedical Visualisation, 200\%. MediVis $200 \%$. International Conference on], 35-42, IEEE (2007).

[17] Wilson, T. A., Legrand, A., Gevenois, P. A., and De Troyer, A., "Respiratory effects of the external and internal intercostal muscles in humans," J Physiol 530, 319-30 (Jan 2001).

[18] Perona, P. and Malik, J., "Scale-space and edge detection using anisotropic diffusion," IEEE Transactions on Pattern Analysis and Machine Intelligence 12, 629-639 (1990). 